\begin{tabular}{|c|l|}
\hline Title & $\begin{array}{l}\text { Active interaction utilizing micro mobile robot and on-line data gathering for experiments in cricket pheromone } \\
\text { behavior }\end{array}$ \\
\hline Author(s) & Kawabata, Kuniaki; A onuma, Hitoshi; Hosoda, Koh; X ue, Jianru \\
\hline Citation & $\begin{array}{l}\text { Robotics and autonomous systems, 61(12), 1529-1538 } \\
\text { https://doi.org/10.1016/.robot.2013.04.021 }\end{array}$ \\
\hline Issue Date & 2013 12 \\
\hline Doc URL & http://hdl.handle.net/2115/54755 \\
\hline Type & article (author version) \\
\hline File Information & RobotA autonSyst_61_final.pdf \\
\hline
\end{tabular}

Instructions for use 


\title{
Active Interaction Utilizing Micro Mobile Robot and On-line Data Gathering for Experiments in Cricket Pheromone Behavior
}

\author{
Kuniaki Kawabata $^{\mathrm{a}, \mathrm{d}, *}$, Hitoshi Aonuma ${ }^{\mathrm{b}}$, Koh Hosodac ${ }^{\mathrm{c}}$, Jianru Xue $^{\mathrm{d}}$ \\ ${ }^{a}$ RIKEN-XJTU Joint Research Team, RIKEN, Saitama, Japan \\ ${ }^{b}$ Research Institute of Electronic Science, Hokkaido University, Hokkaido, Japan \\ ${ }^{c}$ Graduate School of Information Science and Technology, Osaka University, Osaka, \\ Japan \\ ${ }^{d}$ The Institute of Artificial Intelligence and Robotics, Xi'an Jiaotong University, Xi'an, \\ China
}

\begin{abstract}
This paper describes about a prototype system for active interaction experiment to a cricket by utilizing an operated micro mobile robot. It also realize to measure/collect behavior data of the cricket on-line. The behavior selection of the cricket (Gyllus bimaculatus) is influenced by the experience or the context in living environment. In our current research, we are trying to investigate neuronal mechanisms underlying adaptive behavior switching of the cricket based on individual interactions However in conventional biological experiments, the conditions are not maneuverable intentionally. Therefore, we are developing an integrated system with for conducting active interaction experiment and gathering behavior data related to the effect of interactions. The prototype consists of a micro mobile robot as a physical interaction agent, camera and a microphone and a computer. The computer is for commanding to the robot by the operator's input. It also works for recording the data of video sequence, on-line motion tracking and the audio during the experiment. Interaction experiments with the cricket utilizing the prototype system were done. From the results, we could confirm it works well
\end{abstract}

\footnotetext{
*Corresponding address : 2-1, Hirosawa, Wako, Saitama, Japan, 351-0198

Email addresses: kuniakik@riken.jp (Kuniaki Kawabata), aon@es.hokudai.ac.jp (Hitoshi Aonuma), koh.hosoda@ist.osaka-u.ac.jp (Koh Hosoda), jrxue@mail.xjtu.edu.cn (Jianru Xue)
} 
especially for maneuvering experimental conditions and on-line experimental data gathering.

Keywords: Active Interaction Experiment, Pheromone Behavior Initiation, Cricket, Micro Mobile Robot, On-line Experimental Data Gathering

\section{Introduction}

Animals on this planet select their own behavior adaptively based in part of according to their environment in real time. This is attributable to neural circuit network plasticity. Therefore, many studies have sought to clarify high-order motion control in animals through behavioral observation and physiological analysis. Generally, human beings have approximately 100 billion neurons in their huge brains, whereas insects have approximately one million neurons in the whole nervous systems as micro brain. In order to understand how to select the behavior adaptively, it would be better to investigate behavior selection mechanism based on simple nervous system. Therefore, in our current research, for understanding adaptive behavior selection as a dynamic system, we are focusing on insects, in which the relationship between neuronal activities and selected behavior is easy to observe and investigate them. For typical example, Webb [1],[2] have done significant works for modeling neural system of the insect. The works showed a sensory-motor system based on the knowledge of the insect's behavior and response.

Cricket (Gryllus bimaculatus) (Fig.1) shows behavior swiching based on the interactions between individuals and it is representative pheromone behavior in insects[3]. Pheromone behavior emerges when animals detect a particular pheromones. For example, in behavior selection for the fighting behavior of crickets (Fig. 2), nitric oxide (NO) is thought to function as a neuromodulator for extracting a specific behavior program from polymorphic circuits in the brain and that the NO/cyclic guanosine monophosphate (cGMP) cascade plays an important role [4]. Several research results show that some kinds of biogenic amine (octopamine(OA), serotonin(5-HT), etc) play important roles to adjust aggressiveness or sensory efficacy in the insect's nucleuses. Also, based on these internal responses, it is well known that the cricket modified its own behavior selection adaptively in the fighting behavior[5],[6]. The cricket also changes the tendency of behavior selection based on its own experiences or context in the other situations[7]. 
However, it is still unclear how spatio-temporally independent/fragmented knowledge obtained by neurophysiological/ethological research can be integrated as a dynamic system. We consider it is important to alternately repeat synthetic and analytical approaches in which a hypothetical model is simulated based on physiological knowledge and, to verify the results obtained in further physiological experiments [8]. Thus, for understanding adaptive behavior selection mechanism as a dynamic system, we are taking a synthetic approach to compose a dynamic model by connecting biological properties with some hypotheses (Fig. 3) [9]. We call it as synthetic neuroethological approach. Thus, we constructed an adaptive behavior selection model in cricket's fighting behavior inspired by the behavior of the neuromodulator and the substrates in the micro brain[10]. Moreover, we also extended such model with some hypotheses from system synthesis aspect[11]. It was verified that such modeling research result contributed for adaptive cooperation algorithm of the artifacts/robots.[12],[13].

Moreover, in order to gather data or information for more understanding dynamic adaptability of crickets, we started to examine to have interactive experiment using a micro mobile robot[14], [15]. This is an interesting and novel trials related to active interaction to the cricket. For more progress to understand adaptability of the cricket, we are intending to set the experimental condition maneuverable system for gathering additional data related to pheromone behaviors. Therefore, we developed a system for active interaction experiment with the cricket and gathering experimental data of the cricket's pheromone behavior on-line. This paper describes a developed prototype and reports several basic experimental results utilizing the system.

This paper is organized as follows. Section 2 introduces about the cricket 
and our previous works for understanding its adaptive behavior. Section 3 describes our prototype system for active interaction and on-line experimental data gathering. Section 4 also describes the experimental results utilizing our prototype system. Finally, Section 5 presents our conclusions.

\section{Cricket : Gryllus Bimaculatus and Previous Works}

Although crickets (Fig.1) do not form swarms, but rather, are solitary in their activities, they do show some social adjustment behaviors. A pheromone communication between individuals is generally the basis of such behavior adjustments of the insects[7]. While pheromone behaviors of insects was once considered to be "hard-wired" and specific pheromones was thought to cause specific behavior, it is becoming clear that the insect pheromone behavior involves plasticity by modification.

A cricket's body is covered with cuticular substances called cuticular pheromone[16]. As a typical example of the pheromone behavior of the male cricket, a male one encountering another cricket first touches it with its antennae and discriminates its gender, then attempts fighting behavior if it is male. If the loser later encounters the winner or senses male pheromone, it shows avoidance behavior i.e., experiencing defeat causes the cricket to select different behavior for the same stimulus adaptively based on the circumstances. As internal modification in the cricket's brain for such fighting behavior between the male crickets, the NO/cGMP cascade is considered particularly closely related to initiate fighting behavior selection. The pheromone is identified [4] and NO is closely related to pheromone behavior modified by experience $[17]$.

In crickets, social interaction is important factor in determining their behavior. This indicates that the otherwise solitary cricket selects its behaviors on the basis of interactions with other individuals. This forms the basis for other research on crickets. Crickets reared in environments with low-density populations show higher aggression than crickets reared in high-density condition [6]. Populations in environments with densities between high and low (medium) have been observed. It was reported to contain one cricket that continuesto attack other males, while the rest of the males attack less and less often [18] We can also consider this an adaptive behavior that occurs in response to interactions during changes in population density.

Thus, in response to individual interactions, the crickets simultaneously show the adaptive modifications on micro brain level, individual behavior 
level and group behavior level. As our considerations, constructing a model that can explain modifications of both individual and group behaviors of field crickets will shed some light on the adaptive behavior selection mechanisms of this species. Therefore, in our previous work, we constructed an adaptive behavior selection model inspired by the nitric oxide NO/cGMP cascade and OA seen from the physiological and the ethological viewpoints in cricket's fighting behavior[10]. Also extended model with efficacy factor for information processing was composed of knowledges/results obtained by biological research and hypotheses from synthetic aspect[11]. Their consistency with actual behavior of the crickets in each level was confirmed by the computer simulations, qualitatively.

For understanding variety adaptive behavior selection mechanism of cricket, it is required to collect more data or information by setting up experimental (especially, interaction) condition according to the purpose. Therefore, we start to examine to construct such a system which realize active interaction experiment with the cricket. In our previous works[14], [15] motion data analysis was done as off-line process with the handwork. It is not useful to gather the data after many experimental works in practical means. This would be also the issue that take good care in the aspect of biological experiment and it should be stress-free to the cricket in natural situation. Shi et. al. [22] developed a system for analyzing rat's behaviors. This system utilized Support Vector Machine, which is a sort of non-linear machine learning method, with many of the visual features and also take learning process based on such features.

However, one pheromone behavior experiment of the cricket is usually conducted for once and the isolation during several days is required for resetting their internal conditions. This means many number of the individual are tested and the set of their visual features also becomes various ones. Therefore, for our application, the method which is possible to cope with such variety of individuals is required. Then we should improved these subjects of on-line tracking data collection on prototype system. Moreover, previous method requires many complex pre-works for setting-up of tracking process. Therefore, we attempt to implement on-line data measurement/collection based on simple setting-up to our interaction system. 


\section{A System for Active Interaction with the Cricket}

In this section, the prototype system for active interaction and on-line data gathering is described. Our design concept is to realize to interact to the cricket by using operated micro mobile robot and to gather the data related to the behavior of the cricket without adding any artificial things. Because such additional ones would cause any stress to crickets.

\subsection{System Configuration}

The configuration of developed prototype system is shown in Fig. 4. The system consists of control unit and measurement unit and it's configuration is listed in below.

- the arena for the cricket and micro mobile robot;

- Camera and the microphone;

- command transmitter (Infrared(IR) signal) to the micro mobile robot;

- input device for control micro mobile robot;

All input/ouput devices are connected to the computer and implemented image processing and information storing program are executed on the computer. Actual outlook of the prototype system is shown in Fig. 5. Here, in this prototype, we utilize a Macbook Pro (CPU: 2.6[GHz] Core 2 Duo, Memory: $4.0[\mathrm{~GB}]$ ) as the computer and IRTrans [19] (a product of IRTrans GmbH, Germany) infrared module which is connected via USB as a command transmitter. A game operation pad with USB is also utilized for the robot operation. A Logicool HD Pro Webcam C920 [20] is utilized for image/sound capturing as an USB camera with the microphone.

\subsection{Control Unit}

The micro mobile robot platform : Ecobe employed in the experiments for active interactions with the crickets is shown in Fig. 6. Originally designed for an educational robotic competition [21] the robot has a size comparable to that of typical crickets. The robot has dimensions of $18 \times 18 \times 22[\mathrm{~mm}]$ and is driven by two differential wheels, has no sensors except for an infrared receiver used for receiving commands encoded into pulses of infrared light.

The parts of the micro mobile robot : Ecobe which has aluminum body are as follows; 
- Motor : watch micro-stepper motor customized for higher torque;

- Battery : rechargeable $3.7[\mathrm{~V}]$ lithium ion polymer battery (65 [mAh]);

- Control board : each robot equippes with a PIC18LF1220 with 4 [kb] flash memory;

- IR receiver : for receiving control commands $(40[\mathrm{kHz}])$ from the computer;

The micro mobile robot does not equip any external sensors. The list of control commands for the micro mobile robot were pre-set as the form of "Platform ID No. + motion command" in the computer and are sent to the robot platform via IR transmitter (USB-connected to the computer) of the system. Typical commands are moving forward/backward, moving fast forward/backward, turning left/right, changing direction to left/right and so on.

For attracting pheromone behavior from cricket, the micro mobile robot must put the cuticular substances of crickets on its body. In our previous examinations[14], the head part of crickets is enough to initiate the pheromone behavior from cricket. Therefore, we wrap the robot body with the white paper and put the head part of the cricket on the fore side of the micro mobile robot for active interaction experiments (Fig. 7). A maker is also put on the top of the robot body and it is utilized for tracking the trajectory of the mobile robot. Fig. 8 shows an example of top view of the experimental arena.

\subsection{Measurement Unit}

\subsubsection{On-line video recording and trajectory tracking}

As typical observation approach of the cricket's behavior is done by visual one in usual ethological experiment. Moreover, it is a sort of troublesome work to analyze the behavior from the video sequence for the interaction experiment. Therefore, we attempt to implement not only the video capturing/recording function but also trajectory tracking function to both of the cricket and micro mobile robot.

For retaining natural conditions, the cricket must be free from any artificial things. It would cause to give the stress to the cricket. It is an ideal condition however the tracking for free motion of the cricket becomes difficult subject. Because the cricket has no typical template for detecting and 
tracking as body size, body shape even in body color. Therefore, we considered the statistical approach would contribute to the sort of our tracking conditions. Here, we apply particle filter[24], [25] to track the cricket and micro mobile robot color tracking subjects. Particle filter approach can simply realize to track the feature by setting the likelihood function and state transition model. It is practical for on-line tracking application utilizing certain number of the particle which is adjustable based on the experimental setup.

The procedure for tracking based on particle filter with the color of pixel and outlier analysis with location information of the particle is as follows. Here $i=1, \cdots, n$ and $k$ indicate the number for specifying the particle and the captured frame, respectively. $m$ is also a superscript which specify tracking target (cricket or micro mobile robot).

1. capture the image data from the camera.

2. initialize each group-set of the particles and the parameters for tracking the cricket and micro mobile robot and generate the state vector of the particle $\left[{ }^{m} p_{1}^{0},{ }^{m} p_{2}^{0}, \cdots,{ }^{m} p_{n}^{0}\right]$ for each target. Here, $n$ is the population number of each group of the particles.

3. tracking the target by repeating below process for every frame of the image.

(a) estimate each state of the particle based on the dynamical model $Q($.$) with system noise \epsilon$.

$$
{ }^{m} \hat{p}_{i}^{k+1}=Q\left({ }^{m} p_{i}^{k},{ }^{m} \epsilon_{k+1}\right)
$$

(b) evaluate the weight ${ }^{m} w_{i}^{k+1}$ using the color value of the estimated location $\left.C{ }^{m} \hat{p}_{i}^{k+1}\right)$ and the target color ${ }^{m} C_{t g}$.

$$
\left.{ }^{m} w_{i}^{k+1}=R\left({ }^{m} C_{t g} \mid C{ }^{m} \hat{p}_{i}^{k+1}\right)\right)
$$

(c) calculate the probability of each particle based by nomalization.

$$
{ }^{m} \eta_{i}^{k+1}=\frac{{ }^{m} w_{i}^{k+1}}{\sum_{j=1}^{n} m^{m} w_{j}^{k+1}}
$$

(d) renew the state vector $\left[{ }^{m} p_{1}^{k+1},{ }^{m} p_{2}^{k+1}, \cdot^{m} p_{n}^{k+1}\right]$ by sampling with replacement. 
4. evaluate the particles by utilizing outlier analysis method .

5. store the image with/without tracking particles to the video file

6. capture the image data and return to procedure 3 .

Actual procedure 3: particle filter tracking is done by utilizing following functions and definitions. As described above, tracking subject means to track body surface color of the cricket and the marker color on the robot. As a practical implementation, black is for the cricket and red is for the marker on top of the robot. In particle filter approach, it is expected to chase the cricket (black color) and micro mobile robot (red) based on likelihood evaluation. Therefore, we define a following equation to evaluate likelihood ${ }^{m} d_{i}$ using the RGB value on each pixel.

$$
{ }^{m} d_{i}=\sqrt{\left({ }^{m} r_{i}-{ }^{m} R_{t g}\right)^{2}+\left({ }^{m} g_{i}-{ }^{m} G_{t g}\right)^{2}+\left({ }^{m} b_{i}-{ }^{m} B_{t g}\right)^{2}}
$$

Here, ${ }^{m} r_{i},{ }^{m} g_{i}$ and ${ }^{m} b_{i}$ are each RGB value of the pixel on the particle $i$ for target $m .{ }^{m} R_{t g},{ }^{m} G_{t g}$ and ${ }^{m} B_{t g}$ indicate each RGB value of the tracking target $m$ respectively. The function ${ }^{m} w_{i}$ for each particle (pixel) is also expressed following function as a normal distribution by utilizing likelihood measure ${ }^{m} d_{i}$.

$$
{ }^{m} w_{i}=\frac{1}{\sqrt{2 \pi} \sigma} \exp \left(\frac{\left({ }^{m} d_{i}\right)^{2}}{2 \sigma^{2}}\right)
$$

$\sigma$ indicates the distribution value for normal distribution. As the dynamic model of the particle, random-walk model is utilized because the motion of the cricket is fast and its tendency in each direction is not unpredictable one. For the robot, the random-walk model is also used. When the robot is moving, the slippage sometime occurs between the wheel and the ground surface. Also, when the cricket attacks to/pushes the robot, the motion of the robot becomes unpredictable one. Estimated state ${ }^{m} \hat{p}_{i}^{k+1}=$ $\left[{ }^{m} \hat{x}_{i}^{k+1},{ }^{m} \hat{y}_{i}^{k+1},{ }^{m} \hat{u}_{i}^{k+1},{ }^{m} \hat{v}_{i}^{k+1}\right]^{T}$ for each particle is renewed by using the following transition calculation. $\left[x_{i}, y_{i}\right]^{T}$ and $\left[{ }^{m} u_{i},{ }^{m} v_{i}\right]^{T}$ are position vector and velocity vector of the particle, respectively.

$$
{ }^{m} \hat{p}_{i}^{k+1}=Q\left({ }^{m} p_{i}^{k},{ }^{m} \epsilon^{k+1}\right)=\left[\begin{array}{cccc}
1 & 0 & 0 & 0 \\
0 & 1 & 0 & 0 \\
0 & 0 & 1 & 0 \\
0 & 0 & 0 & 1
\end{array}\right]{ }^{m} p_{i}^{k}+{ }^{m} \epsilon^{k+1}
$$


Here, ${ }^{m} \epsilon^{k+1}$ indicates a vector with white random noise. If the position of any particle is set to the out of the image, the position is renewed as new generated one. Moreover, the tracking for the cricket and micro mobile robot must be done simultaneously and be on-line processing. In order to extend above mentioned tracking method to two targets tracking, we set the two group of the particles. On each captured image, the processing for each particle group are done independently.

For procedure 4: outlier analysis which is more accurate tracking by the particle filter, we consider to introduce outlier analysis approach. Mahalanobis distance[26] is a distance measure based on the correlations between variables and it is utilized for cluster analysis and discriminate analysis. Mahalanobis distance is calculated by using the variables, its average value and covariance matrix. Here, we use the position of the particle ${ }^{m} \xi_{i}$ for outlier analysis with Mahalanobis distance (eq. 7) and practical equations are as follows.

$$
\begin{aligned}
{ }^{m} M D_{i} & =\sqrt{\left({ }^{m} \xi_{i}-m \bar{\xi}\right)^{T}{ }^{m} \Xi^{-1}\left({ }^{m} \xi_{i}-m \bar{\xi}\right)} \\
{ }^{m} \xi_{i} & =\left[{ }^{m} x_{i}{ }^{m} y_{i}\right]^{T} \\
{ }^{m} \bar{\xi} & =\frac{1}{n} \sum_{i=1}^{n} \xi_{i} \\
{ }^{m} \Xi & =\frac{1}{n-1} \sum_{i=1}^{n}\left({ }^{m} \xi_{i}-{ }^{m} \bar{\xi}\right)\left({ }^{m} \xi_{i}-{ }^{m} \bar{\xi}\right)^{T}
\end{aligned}
$$

By this calculation, the average value of all ${ }^{m} M D_{i}$ is set to 1.0 and ${ }^{m} M D_{i}=0.0$ means the sample is the center of the cluster. When the sample $i$ takes larger value, there is possibility to be an outlier in the cluster. Here, we can select appropriate threshold for eliminate the outlier particles.

Utilizing above-mentioned calculations, we conducted basic experiments for confirming tracking performance. The target of former one is body color of the cricket (black) and the one of latter one is red circle marker on the micro mobile robot. Here, target colors for tracking are set to black and red in RGB color feature expression as follows.

- $\operatorname{cricket~(black)~:~} m=c:{ }^{c} R_{t g}=0,{ }^{c} G_{t g}=0,{ }^{c} B_{t g}=0$

- micro mobile robot (red) : $m=r:{ }^{r} R_{t g}=255,{ }^{r} G_{t g}=0,{ }^{r} B_{t g}=0$ 
Threshold value for the outlier analysis is set to 2.0. Figure 9 and 10 shows the results for cricket tracking and micro robot tracking, respectively. The center of the circle indicates the average position of each group of the confident particles. The results shows it would work well for each target tracking.

\subsubsection{Audio Recording}

For observation of the crickets' behavior, it is important to gather not only the trajectory or visual information but also audio information. The sound which the cricket makes is a sort of signs which indicate the transition of internal state. Aggressive song by a winner to the defeated one and calling and courtship song are quite different type of the sound. It means that it would be important evidence to understand its transition of attracting certain behavior by active interactions.

In developed prototype system, the microphone in the camera is utilized and on-line recording program is developed by using a free, cross-platform, open-source, audio I/O library : portaudio[27]

\section{Experimental Results}

Utilizing the prototype, we conducts experimential trials to confirm the performance. The operator maneuver the micro mobile robot by seeing the bird eye view of the arena.

\subsection{Behavior Attraction and Video Recording}

Fig.11 shows example snapshots from captured video sequence during single interaction experiment. In this case, a head of male cricket was set in the fore side of the micro mobile robot and the figure shows some part of the sequence in fighting behavior of the male cricket. From several experimental results, we can confirm that the robot could attract the pheromone behaviors including fighting behavior and courtship behavior.

The video recording was implemented by a using open source computer vision library : OpenCV[23]. The video capturing was done by 20 [fps] and captured original video data and the one with the particles are stored in the storage.

\subsection{Tracked Trajectories}

The tracking process by particle filter was also implemented by a using open source computer vision library : OpenCV[23]. The image size for experiments was set to $640 \times 480$ [pixel] and actual arena size was $200[\mathrm{~mm}] \times$ 
$150[\mathrm{~mm}]$. Tracking process with proposed above was conducted on 20 [fps]. The color values of target for tracking are also similar setting with basic tracking experiment (for cricket : $m=c ;{ }^{c} R_{t g}=0,{ }^{c} G_{t g}=0,{ }^{c} B_{t g}=0$, and for micro mobile robot: $\left.m=r ;{ }^{r} R_{t g}=255,{ }^{r} G_{t g}=0,{ }^{r} B_{t g}=0\right)$. The number of the particle for tracking the cricket was 300 and the one for the robot was 200 . Noise value on random-walk model for the position vector of each particle was selected between -25 and 25[pixel. The one for the velocity vector of each particle was set between -5 and 5 [pixel]. Threshold value for the outlier analysis is set to 2.0 .

Fig.12 shows the snapshots from the video sequence with tracked particles. Fig. 13 also shows the trajectory data which are stored on-line processing during the interaction experiments. Upper figure is to plot both of the trajectories of the cricket and the micro mobile robot. Lower ones indicate each trajectory. $S_{C}, F_{C}, S_{R}$ and $F_{R}$ in the figures indicate initial / final location of the cricket and the ones of the micro mobile robot, respectively. The source of this video sequence was also the same one with Fig.11. In sometime during this video sequence, each swarm of the particles is closed or overlapped to each other however both of the cricket and the robot were discriminated correctly in such case. Moreover, Figure 14 shows an example of the interaction experiment between male cricket and the operated mobile robot with female cricket. Figure 15 indicates on-line tracking result during this experiment.

As for the evaluation method to the tracking results, we took visual inspection to recorded data with the tracking result (like Fig. 12 and 14). The inspector checked the tracking results were not out of the cricket's body and the marker on the robot. We could confirm the tracking was done in several experimental trials with recorded video correctly. The original/tracked video data and tracked trajectory data in 2-D space are stored on the storage of the computer on-line.

\subsection{Audio Recording}

Sound data is also recorded in the format of stereo raw type digital data $(44100 \mathrm{~Hz}, 32 \mathrm{bit})$. Implemented program utilizing portaudio stores captured data on-line in the hard disk of the computer. We confirm to hear the data after the experiments and it is enough quality to recognize the cricket's song. 


\section{Conclusions}

It is known that the cricket shows adaptive behavior selection based on interaction with the other. Investigating such behavior selection mechanism is useful for not only scientific aspect but also engineering/system design aspect. In order to progress to understand such mechanism as a dynamic system, behavior experiment setup with control interaction and collect behavior data is required. Therefore, in this paper, we developed and described a prototype system for active interaction experiment with a cricket.

By utilizing developed prototype system, the operator can maneuver the micro mobile robot and set up the interaction condition with the cricket intentionally. The system also could realize to gather the behavior data of the cricket on-line. Video sequences during the experiment are recorded online. Trajectory tracking for the cricket and the robot is also realized by utilizing particle filter approach and outlier analysis. Audio data which is a typical indicator of internal state transition of the cricket is also recorded on-line. Utilizing the prototype system, we conducted several experiments and confirmed it works well for active interaction experiments and on-line data gathering (video, tracker and sound).

As our future work, we would develop orientation estimation for both of the cricket and mobile robot. Also, we would design command sequences for controlling the robot behavior for automatic interaction experiment based on on-line data gathering. We are also now considering about a measuring method for the cricket's internal conditions related to the chemical substances. It would be integrated for realizing on-line multiple channel state recording system.

\section{Acknowledgement}

This work has been partially supported by KAKENHI ( No. 23650102 and No. 23300113) from the Japanese Ministry of Education, Culture, Sports, Science and Technology and NSFC project 90920301 and and 61273252 of China. We also thanks Dr. R. S. Guerra on his helpful advise related to the fundamental set-up for micro mobile robot operation. 


\section{References}

[1] Webb, B., "Robots, crickets and ants: models of neural control of chemotaxis and phonotaxis.", Neural Networks, vol. 11, no. 7-8, pp. 1479-1496, Oct. 1998.

[2] Webb, B., "Robots in invertebrate neuroscience.", Nature, vol. 417, no. 6886, pp. 359-63, May 2002.

[3] Regnier, Fred E. and Law, John H. "' Insect pheromones ” , Journal of Lipid Research, 9:(5) 541-551, 1968.

[4] Aonuma, H. , and Niwa, K. ,“ Nitric Oxide Regulates the Levels of cGMP Accumulation in the Cricket Brain," Acta Biologica Hungarica, 55, pp. 65-70, 2004.

[5] Sakura, M., Yoritsune, A. , and Aonuma, H. ," Fighting experiences modulate aggressive and avoidance behaviors in crickets against male cuticular substances, The 2nd International Symposium on Mobilligence, pp243-246, 2007.

[6] Ashikaga, M. , Sakura, M. , Kikuchi, M. , Hiraguchi, T. , Chiba, R. , Aonuma, H. and Ota, J., "Establishment of social status without individual discrimination in the cricket", Advanced Robotics, Vol.23, No.5, pp.563-578, 2009.

[7] Alexander, R. D.," Aggressiveness, Territorialiality, and Sexual Behavior in Field Crickets (ORTHOPTERA : GRYLLIDAE), Behaviour, Vol. 17, No. 2-3, pp130-223, 1961.

[8] Asama, H. , "Mobiligence: Emergence of Adaptive Motor Function through Interaction among the Body, Brain and Environment," Proceedings of International Conference on Mechatronics and Automation 2007, pp.31-32, 2007.

[9] Aonuma, H. , and Kanzaki, R.," Systematic Understanding of Neuronal Mechanisms for Adaptive Behavior in Changing Environment," Proc. of the 1st Int. Symposium on Mobiligence, pp. 63-66, 2005.

[10] Kawabata, K. , Fujiki, T. , Ikemoto, Y. , Aonuma, H. , Asama, H. ,“ A Neuromodulation Model for Adaptive Behavior Selection by the 
Cricket - Nitric Oxide (NO)/Cyclic Guanosine MonoPhosphate (cGMP) Cascade Model “ , Journal of Robotics and Mechatronics, Vol. 19, No.4, pp.388-394, 2007.

[11] Kawabata, K. , Fujii, T. , Aonuma, H. , Suzuki, T. , Ashikaga, M. , Ota, J. , and Asama, H.," A Neuromodulation Model of Behavior Selection in the Fighting Behavior of Male Crickets" , Robotics and Autonomous Systems, Vol. 60, Issues 5, pp.707-713, 2012.

[12] Asikaga, M., Kikuchi, M., Hiraguchi, T., Sakura, M., Aonuma, H., Ota, Jun. "Foraging Task of Multiple Mobile Robots in a Dynamic Environment Using Adaptive Behavior in Crickets", Journal of Robotics and Mechatronics, Vol.19, No.4, pp466-473, 2007.

[13] Kawabata, K., Fujii, T., Suzuki, T., Aonuma, H., Ota, J., Asama, H., "Sweeping Task of Multiple Mobile Agents by Utilizing Behavior Selection Model with Interaction-Based Efficacy Dynamics", ransactions of the Japan Society of Mechanical Engineers, Series (C), Vo. 78, No. 792, pp3028-3032, 2012 (in Japanese)

[14] Guerra, R. S., Aonuma, H., Hosoda, K., Asada, M., : “ Semi-automatic behavior analysis using robot/insect mixed society and tracking”, Journal of Neuroscience Methods, 191, pp138-144, 2010.

[15] Guerra, R. S., Aonuma, H., Hosoda, K., Asada, M., :“ Behavior Change of Crickets in a Robot-Mixed Society" , Journal of Robotics and Mechatronics, Vol.22, No.4, pp. 526-531,2010.

[16] Nagamoto, J., Aonuma, H. and Hisada, M.: "Discrimination of Conspecific Individuals via Cuticular Pheromones by Males of Cricket Gryllus bimaculatus," Zoological Science, 22, pp.1079-1088, 2005.

[17] Matsumoto, Y. , Unoki, S. , Aonuma, H. , and Mizunami, M. ,“ Nitric Oxide-cGMP Signaling is Critical for cAMP-dependent Long-term Memory Formation," Learning \& Memory, 13(1), pp. 35-44, 2006.

[18] FunatoT., Nara M., Kurabayashi D., Ashikaga M. and Aonuma H. A model for groupsize-dependent behaviour decision in insect using an oscillator network. J. Exp. Biol.214: 2426-2434, 2011.

[19] http://www.irtrans.com/ (May. 2012) 
[20] http://www.logicool.co.jp/ja-jp/webcam-communications/webcams/devices/9442 (May. 2012)

[21] Guerra, R. S. , Boedecker, J. , Yanagimachi, S. , Ishiguro, H. and Asada, M. "“ A New Minirobotics System for Teaching and Researching Agentbased Programming," In V. Uskov, editor, Proc. of Computers and Advanced Technology in Education - 2007, October 2007.

[22] Shi, Q., Miyagishima, S., Fumino, S., Konno, S., Ishii, H. and Takanishi, A. , "Development of a cognition system for analyzing rat's behaviors," Proceedings of IEEE International Conference on Robotics and Biomimetics, pp. 1399-1404, 2010.

[23] http://opencv.willowgarage.com/wiki/ (May. 2012)

[24] Ristic, B. , Arulampalam, S. , Gordon, N. "“ Beyond the Kalman Filter: Particle Filters for Tracking Applications” , Artech House, 2004.

[25] Isard, M. , and Blake, A. ,“ CONDENSATION - conditional density propagation for visual tracking" , International Journal of Computer Vision, 29, 1, 5-28, 1998.

[26] Mahalanobis, P. C. , On the generalised distance in statistics, Proceedings of the National Institute of Sciences of India, 2, 49-55, 1936.

[27] http://www.portaudio.com/ (Mar. 2012) 


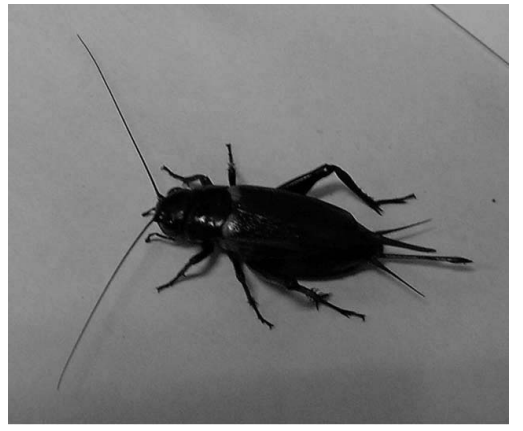

Figure 1: Female cricket : Gryllus bimaculatus
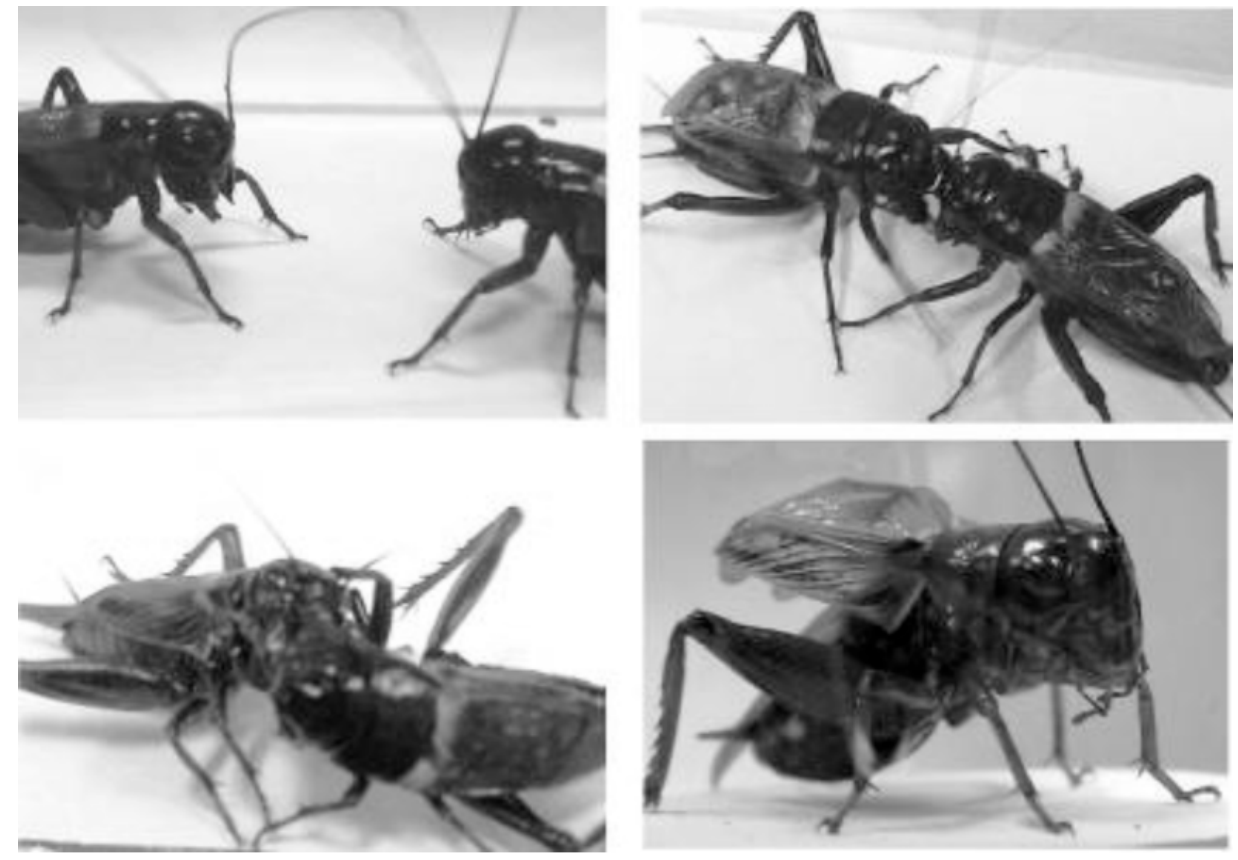

Figure 2: Snapshots of fighting behaviors between male crickets 


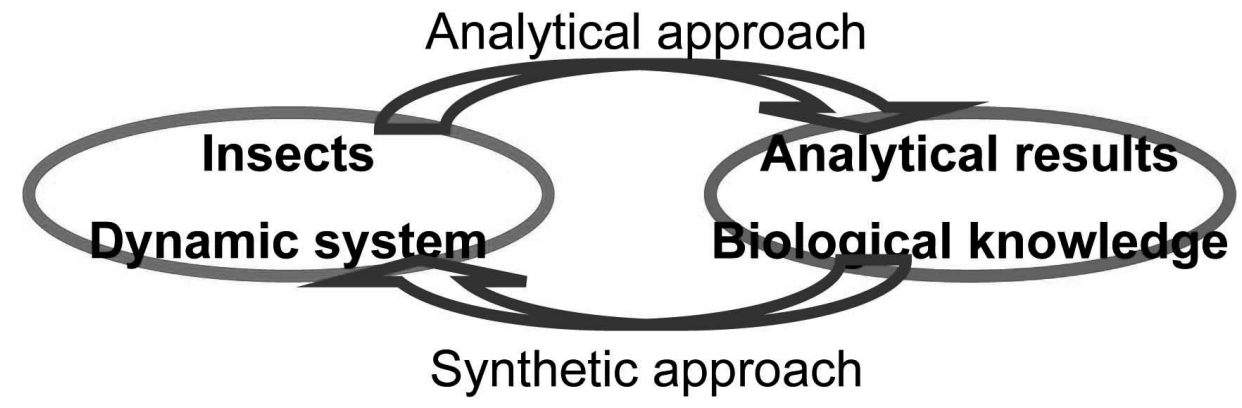

Figure 3: Approach of our current research for understanding adaptive behavior selection mechanism

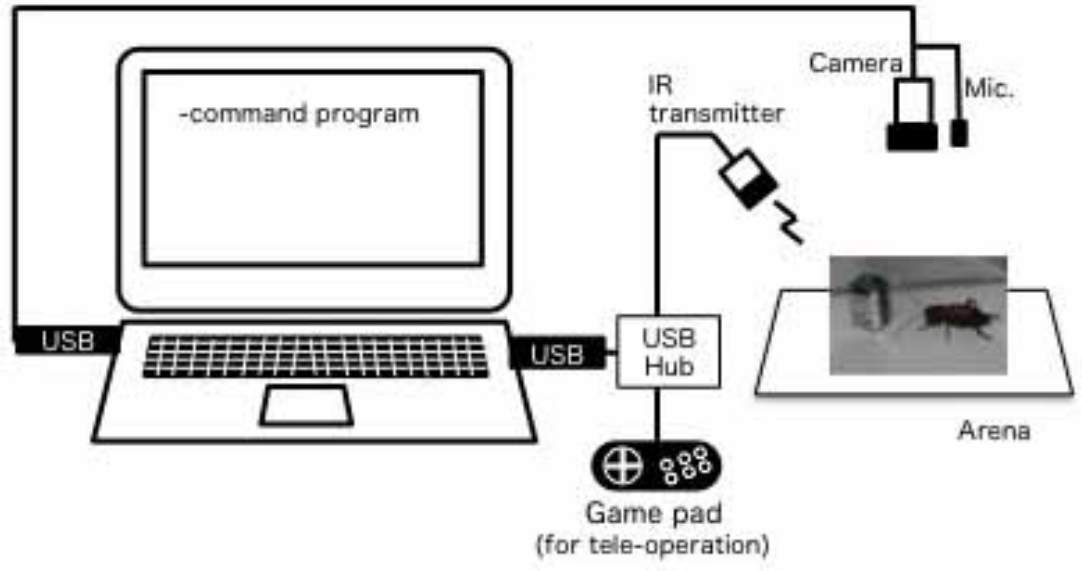

Figure 4: System configuration of the experimental prototype 


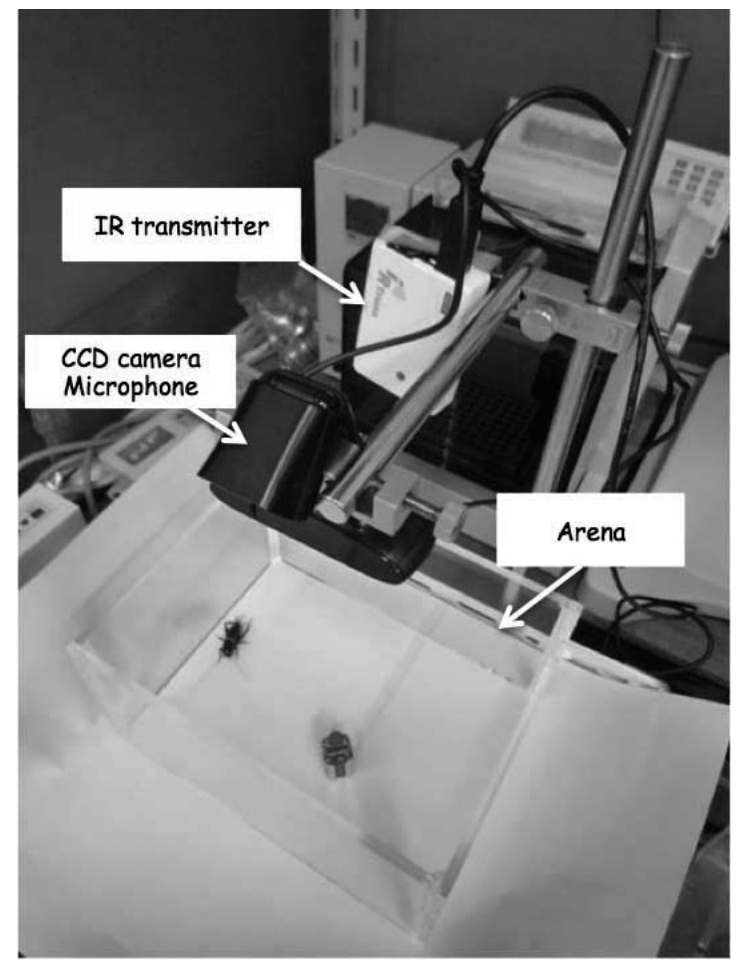

Figure 5: Outlook of the experimental system

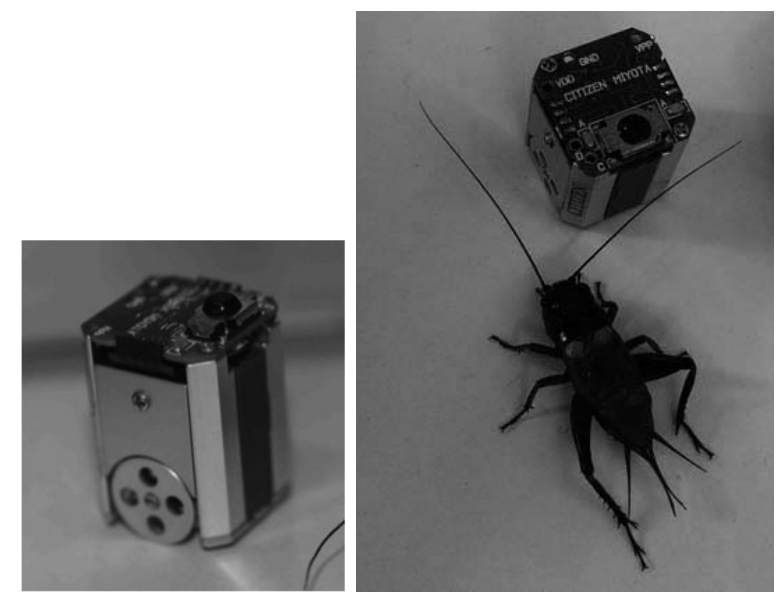

Figure 6: Ecobe : a micro mobile robot 


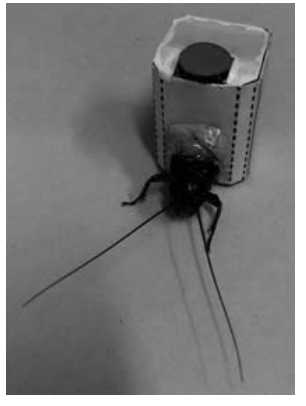

Figure 7: Outlook of the experimental setup of micro mobile robot

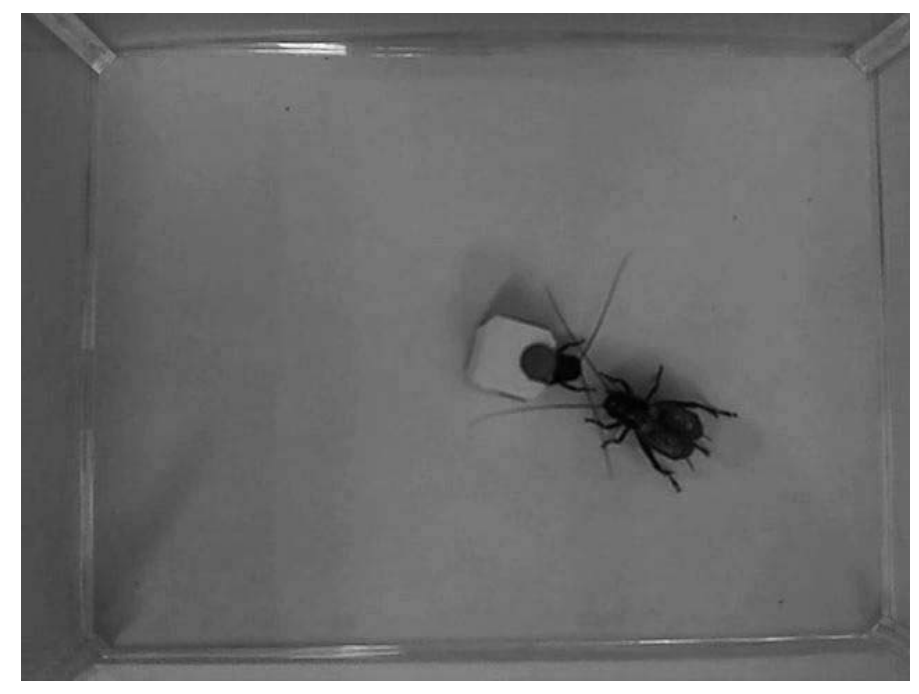

Figure 8: Top view of interaction experiment 

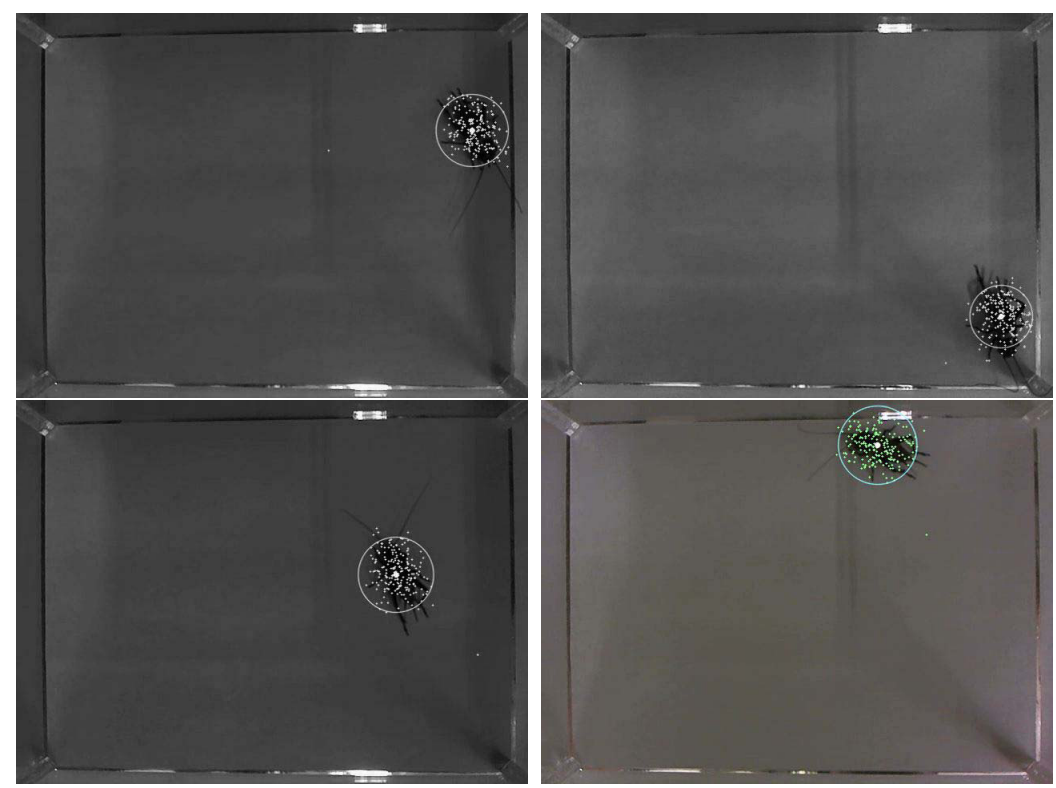

Figure 9: Snapshots of tracking a cricket

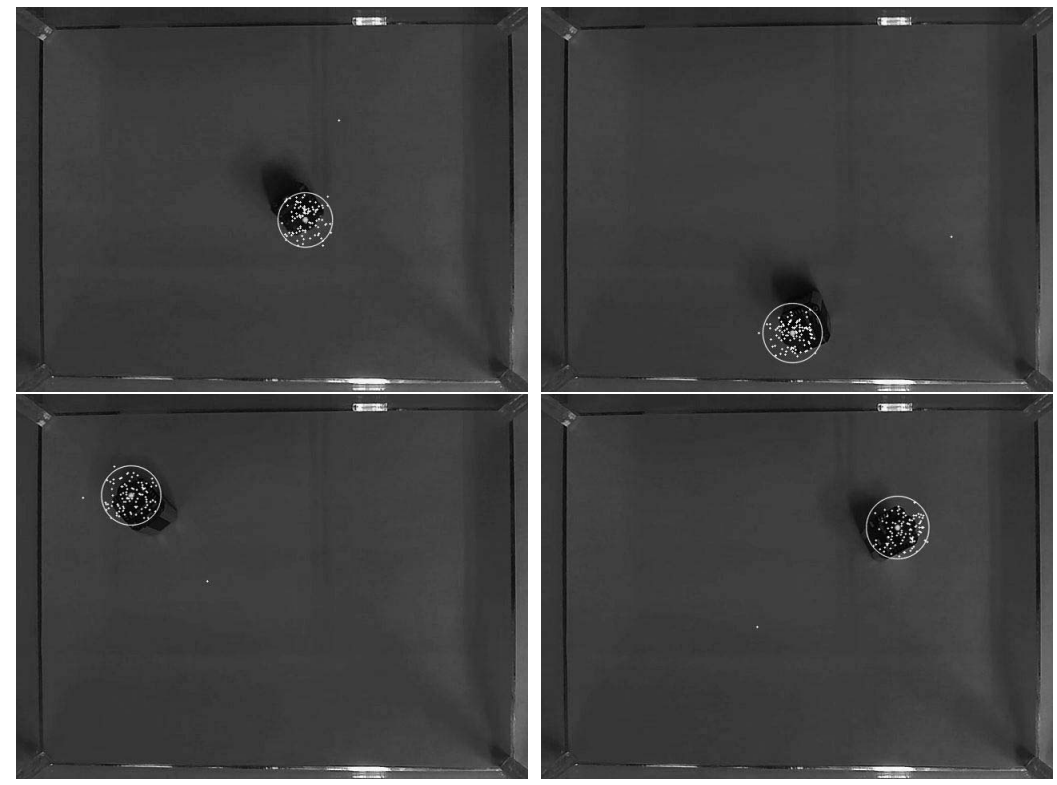

Figure 10: Snapshots of tracking a micro mobile robot 

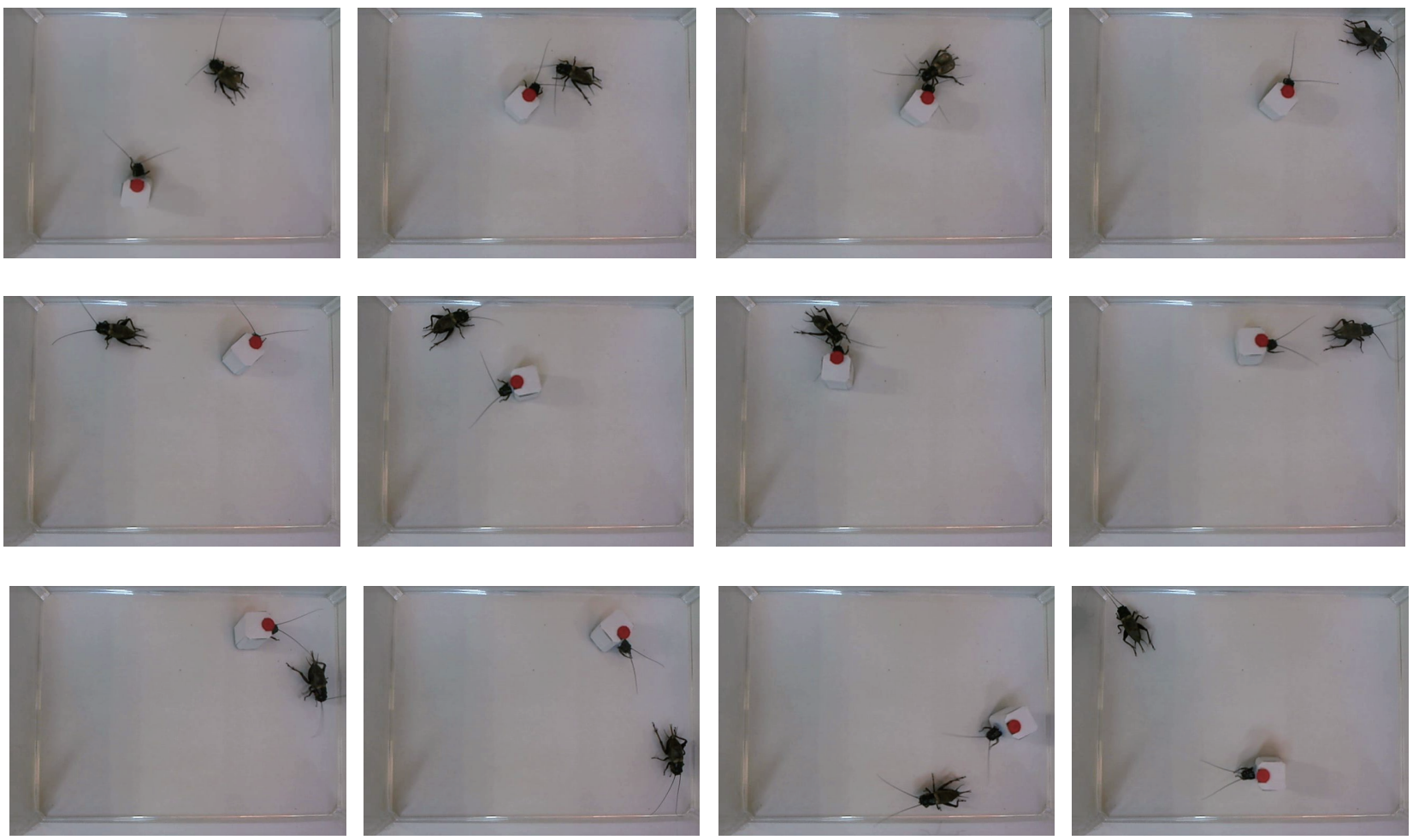

Figure 11: Snapshots of fighting behaviors between male cricket and micro mobile robot with male head 

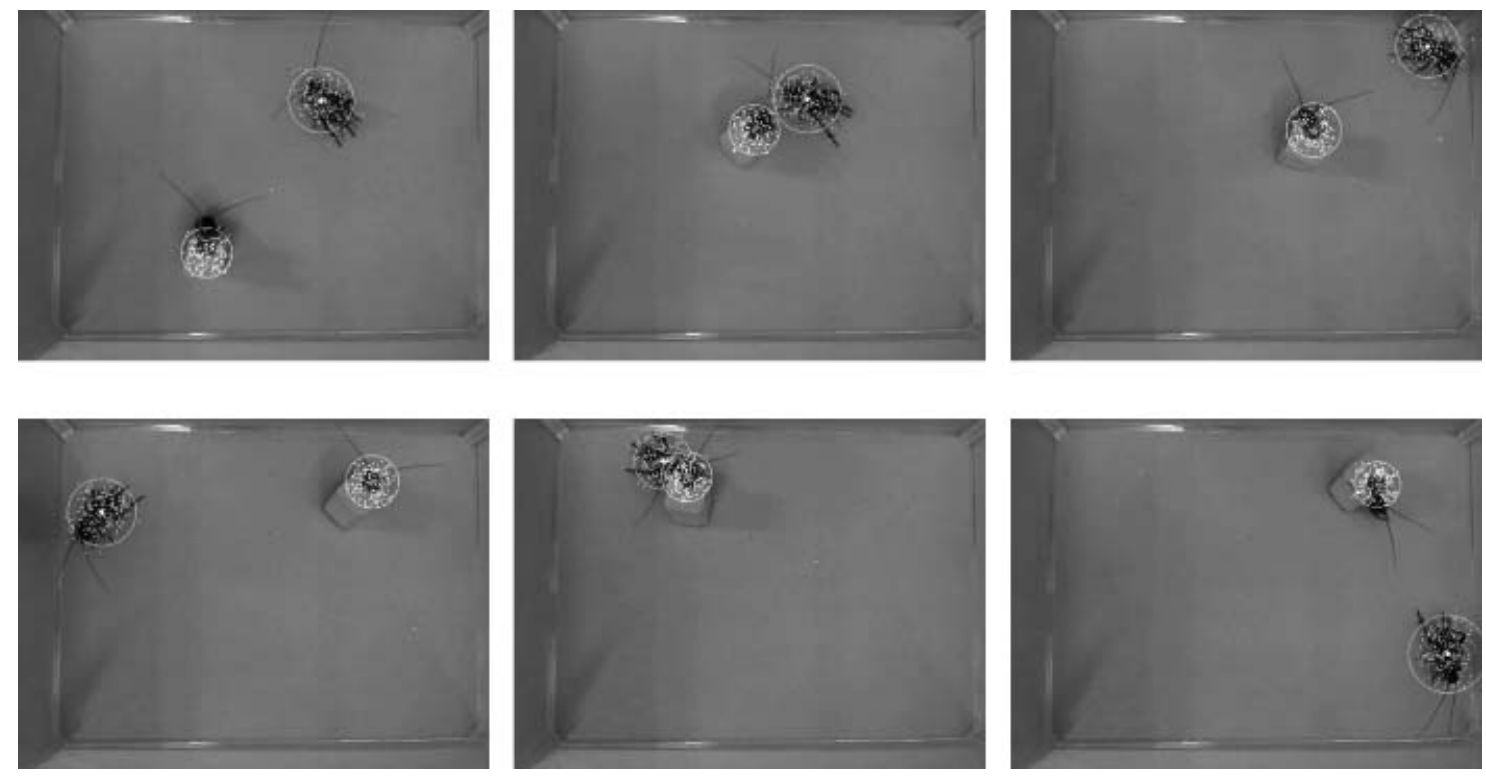

Figure 12: Snapshots of tracking processing by using particle filter 

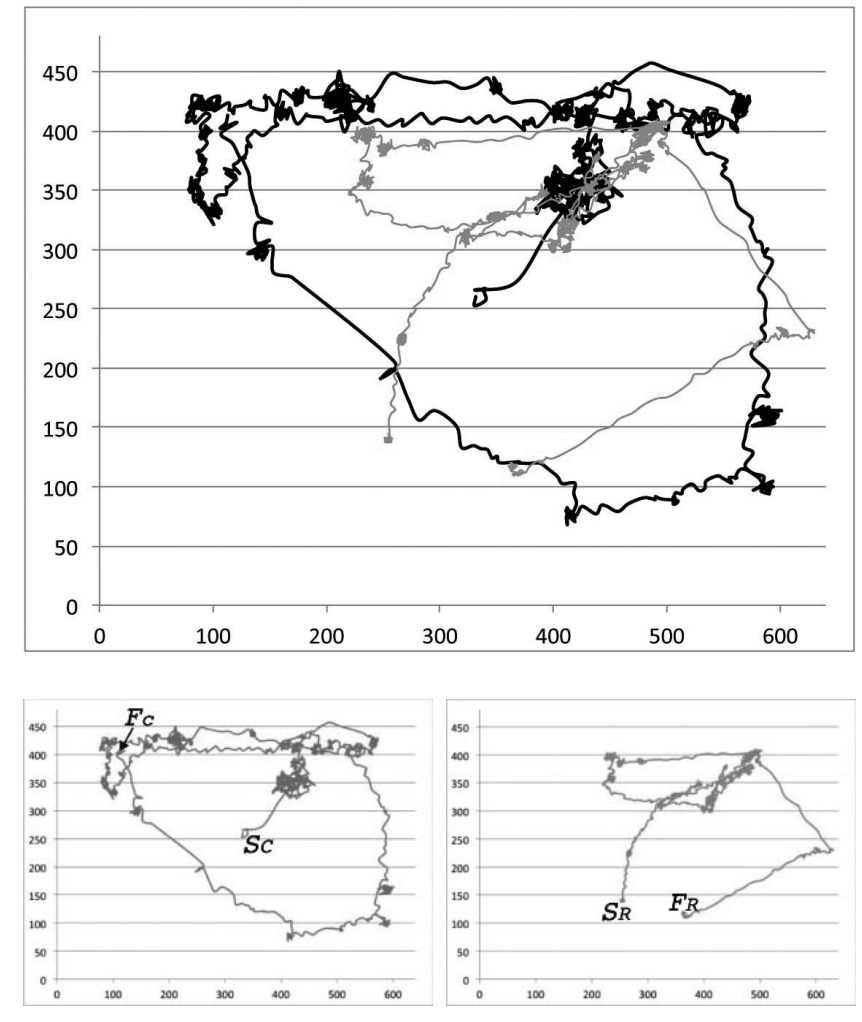

Figure 13: Tacked trajectories(upper). Independent trajectory of the male cricket (below left) and the micro mobile robot with male head(below right) 

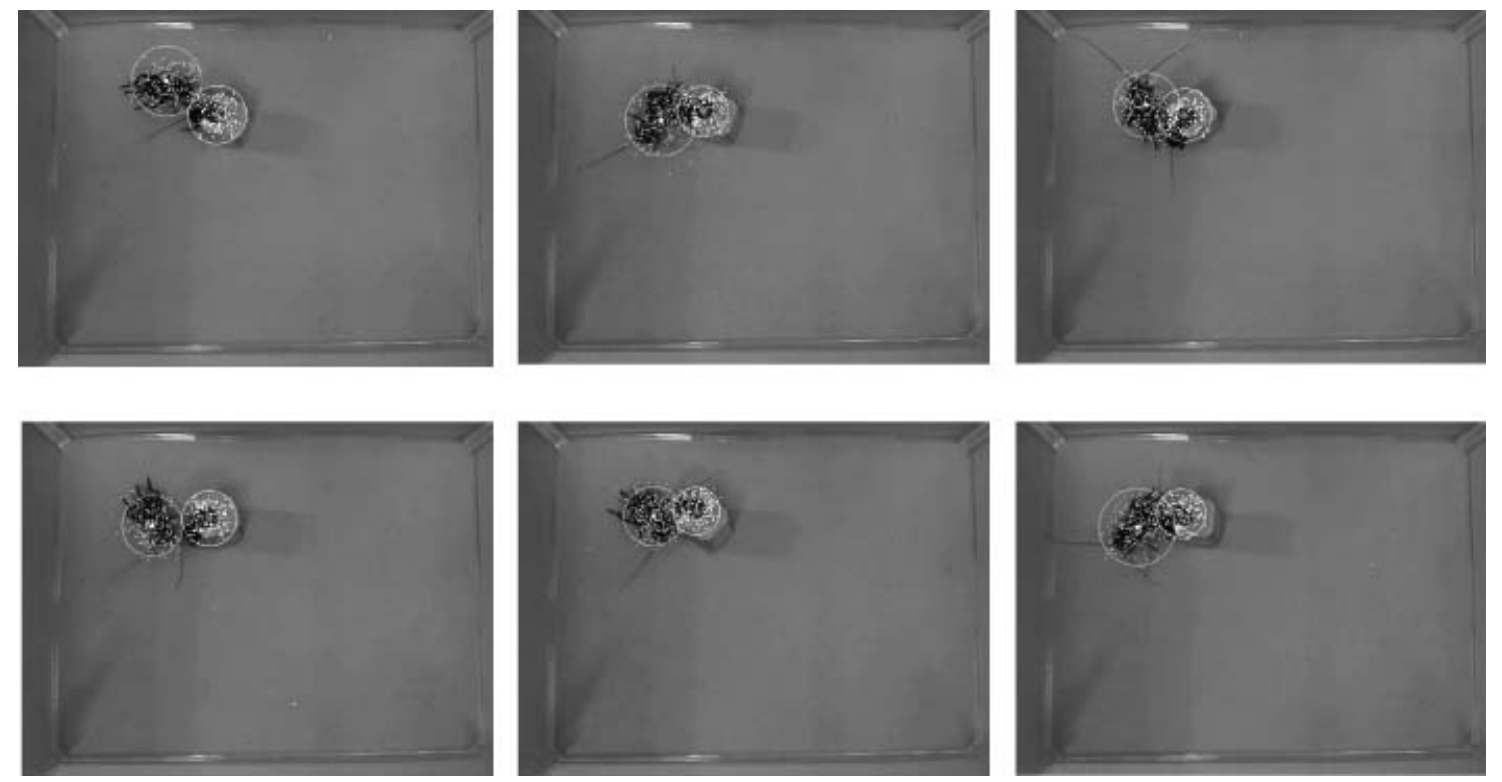

Figure 14: Snapshots of tracking processing by using particle filter 

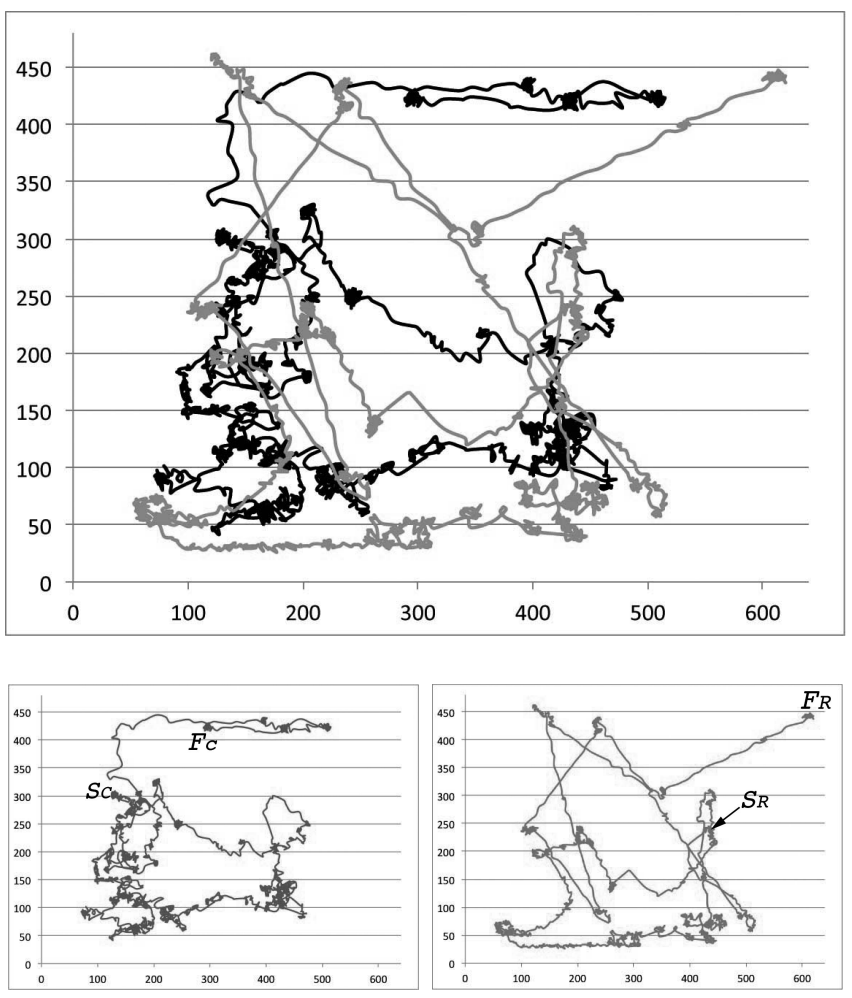

Figure 15: Tacked trajectories(upper). Independent trajectory of the male cricket (below left) and the micro mobile robot with female head(below right) 